\title{
Weak Exponential Stability for Time-Periodic Differential Inclusions via First Approxima- tion Averaging
}

\author{
R. Gama and G. Smirnov
}

\begin{abstract}
In this work we propose a method to study a weak exponential stability for time-varying differential inclusions applying an averaging procedure to a first approximation. Namely, we show that a weak exponential stability of the averaged first approximation to the differential inclusion implies the weak exponential stability of the original time-varying inclusion. The result is illustrated by an example.
\end{abstract}

Mathematics Subject Classification (2010). Primary 34A60; Secondary $34 \mathrm{C} 29$.

Keywords. Differential inclusions, Averaging method.

\section{Introduction}

The study of stability proprieties of nonautonomous nonlinear systems is known as a hard problem. One of the techniques used to simplify the consideration is the averaging method consisting in replacing the original system by an averaged autonomous one which has similar proprieties but its analysis is easier.

Several results relating exponential stability proprieties of the averaged and original differential equations can be already found in the classical book by Bogoliubov and Mitropolski [2]. Bogoliubov and Mitropolski used classical linearization of the averaged differential equation to formulate the sufficient conditions of exponential stability for the original equation. Later, in [7, 11], under some homogeneity conditions, it was shown that the asymptotic stability properties of the original equation can be derived from the respective asymptotic stability properties of the averaged equation. In $[1,6,9]$ similar results were established without any homogeneity conditions.

This work was completed with the support of our $\mathrm{T}_{\mathrm{E}} \mathrm{X}$-pert. 
The averaging method was also successfully used to solve some control problems. For example, in [8], it was applied to construct an explicit timevarying feedback laws for attitude stabilization of a spacecraft subject to twodimensional control. Recently, in [5], the authors studied weak exponential stability of time-varying linear control systems and applied their results to prove the existence of almost closed relative trajectories for satellite formation flying with single-input control.

In this paper we study weak exponential stability of differential inclusions via its first approximation averaging. Our approach differs from the classical one $[2,3]$, where stability properties of the original system are obtained analyzing the first approximation of the averaged system. The averaging of the first approximation allows us to get easy verifiable sufficient exponential stability conditions and to make the proof more natural and direct. Some other results concerning weak stability for differential inclusions via the averaging method can be found in the survey [10].

The paper is organized as follows. In Section 2 some background mathematical results, used later on, are gathered. We present our main result in Section 3, establishing exponential stability for a time-varying differential inclusion applying the averaging method to the respective first approximation. In Section 4, the obtained results are applied to differential inclusions generated by control systems. This section also contains an illustrative example.

Throughout this paper we denote by $\mathbb{R}^{n}$ the real $n$-dimensional space and by $|\cdot|$ the usual Euclidean norm, respectively. We use the notation $B_{n}=\left\{x \in \mathbb{R}^{n}|| x \mid \leqslant 1\right\}$ for the closed unit ball in $\mathbb{R}^{n}$. The Minkowsky function of a set $A \subset \mathbb{R}^{n}$ is denoted by $\mu(x)=\inf \{\alpha>0 \mid x / \alpha \in A\}$. The notation $\mathcal{F}\left(\mathbb{R}^{n}\right)$ is used for the set of all closed subsets from $\mathbb{R}^{n}$. The interior, the convex hull, and the closure of a subset $S \subset \mathbb{R}^{n}$ are denoted by int $S$, $\operatorname{co} S$, and $\operatorname{cl} S$, respectively. We denote by $\mathcal{S}_{[0, T]}(F, C)$ the set of solutions to differential inclusion $\dot{x} \in F(t, x), t \in[0, T]$, satisfying $x(0) \in C \subset \mathbb{R}^{n}$. The closed unit ball in the space of continuous functions, $C\left(\left[0,+\infty\left[, \mathbb{R}^{n}\right)\right.\right.$, with the uniform norm, is denoted by $\mathcal{B}$. The graph of a set-valued map $F: X \rightarrow Y$ is denoted by $\operatorname{gr} F=\{(x, y) \in X \times Y \mid y \in F(x)\}$, and its domain is defined as $\operatorname{dom} F=\{x \in X \mid F(x) \neq \emptyset\}$.

\section{Preliminary Notes and Results}

To make the paper self-contained we include in this section some background material used to state and prove our main result. Consider a differential inclusion

$$
\dot{x} \in F(t, x),
$$

and assume that the following conditions are satisfied:

(C1) $0 \in F(t, 0), \forall t \geqslant 0$;

(C2) The map $F$ is periodic in $t$ with the period $T>0$, i.e., $F(t+T, x)=$ $F(t, x)$ for all $(t, x) \in \mathbb{R} \times \mathbb{R}^{n}$; 
(C3) The map $F(\cdot, x)$ is measurable for all $x \in \mathbb{R}^{n}$. There exists $b(\cdot) \in$ $L_{1}([0, T], \mathbb{R})$ such that $F(x, t) \subset b(t) B_{n}$ for all $(t, x) \in \mathbb{R} \times \mathbb{R}^{n} ;$

(C4) The map $F(t, \cdot)$ is Lipschiztian for all $t \in[0, T]$, with a constant $k(t)$, where $k(\cdot) \in L_{1}([0, T], \mathbb{R})$, i.e. $F\left(t, x_{1}\right) \subset F\left(t, x_{2}\right)+k(t)\left|x_{2}-x_{1}\right| B_{n}$ for all $x_{1}$ and $x_{2}$ in $\mathbb{R}^{n}$ and $t \in[0, T]$.

Recall the definitions of weak stability.

Definition 2.1. The equilibrium position $x=0$ of differential inclusion (2.1) is said to be weakly asymptotically stable if, given any $\epsilon>0$, there exists $\delta>0$ such that for any $x_{0} \in \delta B_{n}$, at least one solution $x(\cdot)$ of $(2.1)$ with $x(0)=x_{0}$ satisfies $|x(t)|<\epsilon$ for all $t \geq 0$, and

$$
\lim _{t \rightarrow \infty} x(t)=0
$$

Definition 2.2. We say that the zero equilibrium position of differential inclusion (2.1) is weakly exponentially stable, if there exist positive constants $c, \gamma$, and $\delta$ such that for any $x_{0} \in \delta B_{n}$ at least one trajectory $x(\cdot)$ of $(2.1)$ with $x(0)=x_{0}$ satisfies

$$
|x(t)| \leqslant c\left|x_{0}\right| e^{-\gamma t}, \quad t \geq 0 .
$$

Weak exponential stability of inclusion (2.1) can be studied through its first approximation. We say that a set-valued map $A: \mathbb{R} \times \mathbb{R}^{n} \rightarrow \mathcal{F}\left(\mathbb{R}^{n}\right)$, with $\operatorname{dom} A(t, \cdot)=\mathbb{R}^{n}, \forall t \geqslant 0$, is a first approximation of the set-valued map $F: \mathbb{R} \times \mathbb{R}^{n} \rightarrow \mathcal{F}\left(\mathbb{R}^{n}\right)$ at the equilibrium position $x=0$, if $A(t, \cdot)$ is a convex process, i.e. $\operatorname{gr} A(t, \cdot)$ is a closed convex cone, and for any $\left(x_{0}, v_{0}\right) \in \operatorname{gr} A(t, \cdot)$ the following equality holds:

$$
\lim _{h \downarrow 0} h^{-1} d\left(h v_{0}, F\left(t, h x_{0}\right)\right)=0,
$$

for all $t \geqslant 0$.

If a first approximation is given we can consider the differential inclusion of first approximation

$$
\dot{x} \in A(t, x) .
$$

We assume that $A$ satisfies the following conditions:

(C5) The map $A(t, \cdot)$ is a first approximation of the map $\operatorname{coF}(\mathrm{t}, \cdot)$ at $x=0$ for all $t$;

(C6) The map $A(\cdot, x)$ is periodic in $t$ of period $T>0$, i.e, $A(t+T, x)=A(t, x)$ for all $(t, x) \in \mathbb{R} \times \mathbb{R}^{n}$;

(C7) The map $A(\cdot, x)$ is measurable for all $x \in \mathbb{R}^{n}$ and $\operatorname{dom} \mathrm{A}(\mathrm{t}, \cdot)=\mathbb{R}^{\mathrm{n}}, \forall \mathrm{t} \geqslant$ 0 ;

In [12], it was shown that under these assumptions the equilibrium position $x=0$ of differential inclusion (2.1) is weakly asymptotically stable whenever the zero equilibrium position of differential inclusion (2.2) is a weakly asymptotically stable. The same proof also leads to the following stronger result. 
Theorem 2.3. Assume that the equilibrium position $x=0$ of differential inclusion (2.2) is weakly asymptotically stable. Then $x=0$ is a weakly exponentially stable equilibrium position of differential inclusion (2.1).

Recall a generalization of the Samoilenko-Stanzhitskii Theorem obtained in [4]. Consider a differential inclusion

$$
\dot{x} \in \epsilon F(t, x),
$$

where $\epsilon>0$ is a small parameter, and the respective averaged inclusion

$$
\dot{\bar{x}} \in \epsilon \bar{F}(\bar{x})=\epsilon \bigcap_{\delta>0} \bar{F}^{\delta}(x)
$$

where $\bar{F}^{\delta}(x)$ is a convex hull of the map

$$
\bar{\Phi}^{\delta}(x)=\limsup _{\theta \uparrow 1} \limsup _{T \rightarrow \infty} \frac{1}{(1-\theta) T} I(\theta T, T, x, \delta),
$$

and

$$
I\left(t_{1}, t_{2}, x, \delta\right)=\left\{\int_{t_{1}}^{t_{2}} v(t) d t \mid v(\cdot) \in L_{1}^{\mathrm{loc}}\left(\left[0, \infty\left[, \mathbb{R}^{n}\right), v(t) \in F\left(t, x+\delta B_{n}\right)\right\} .\right.\right.
$$

(The limsup stands for the Kuratowski upper limit, i.e., the set of all limit points.) It is assumed that the following conditions are satisfied:

(H1) $\operatorname{clco} F(t, x)=F(t, x)$, for all $(t, x) \in \mathbb{R} \times \mathbb{R}^{n}$;

(H2) the set-valued map $F(t, \cdot)$ is upper semi-continuous;

(H3) for any $x$ there exists a measurable selection of $F(t, x)$, i.e., a measurable function $t \rightarrow f(t, x)$ satisfying $f(t, x) \in F(t, x)$;

(H4) there exists a nonnegative $b(\cdot) \in L_{1}^{\text {loc }}([0, \infty[, \mathbb{R})$ such that $F(t, x) \subset$ $b(t) B_{n}$ for all $(t, x) \in\left[0,+\infty\left[\times \mathbb{R}^{n}\right.\right.$

(H5) there exists the limit

$$
b=\lim _{T \rightarrow \infty} \frac{1}{T} \int_{0}^{T} b(t) d t
$$

Under these assumptions the following theorem holds true.

Theorem 2.4. Let $F: \mathbb{R} \times \mathbb{R}^{n} \rightarrow \mathbb{R}^{n}$ be a set-valued map satisfying conditions (H1) - (H5). Assume that $\bar{x}=0$ is an asymptotically stable equilibrium position of the differential inclusion $\dot{x} \in \epsilon \bar{F}(x)$. Then for any $\eta>0$ there exist $\epsilon_{0}>0$ and $\delta>0$ such that $\mathcal{S}_{[0, \infty[}(\epsilon F, \delta B) \subset \eta \mathcal{B}$, whenever $\left.\epsilon \in\right] 0, \epsilon_{0}[$.

Thus the asymptotically stability of the equilibrium position of the averaged inclusion implies the boundedness of the original system solutions.

\section{Weak exponential stability for differential inclusions}

In this section we show that weak exponential stability of the zero equilibrium position of the differential inclusion

$$
\dot{x} \in \epsilon F(t, x),
$$


can be inferred from the weak asymptotic stability of its averaged first approximation. We assume that the set-valued map $F$ satisfies conditions (C1)(C4). Let $A: \mathbb{R} \times \mathbb{R}^{n} \rightarrow \mathcal{F}\left(\mathbb{R}^{n}\right)$ be a set-valued map satisfying conditions (C5)-(C7).

Define the averaged convex process as

$$
\bar{A}(\bar{x}):=\left\{\frac{1}{T} \int_{0}^{T} a(s, \bar{x}) d s \mid a(s, \bar{x}) \in A(s, \bar{x}), a(s, \bar{x}) \in L_{1}\left([0, T], \mathbb{R}^{n}\right)\right\},
$$

and consider the associated averaged differential inclusion

$$
\dot{\bar{x}} \in \epsilon \bar{A}(\bar{x})
$$

Several results concerning weak asymptotic stability of autonomous differential inclusions like (3.2) are known. Let $\bar{x}=0$ be a weakly asymptotically stable equilibrium position of (3.2). From [13, Theorem 9.1] we see that there exist a convex polyhedron $\mathfrak{M}$, with the vertices $\left\{\bar{x}_{1}, \ldots, \bar{x}_{m}\right\}$, and numbers $h>0$ and $\delta \in] 0,1[$ such that $0 \in$ int $\mathfrak{M}$, and for every $k=\overline{1, m}$ there exists a vector $\bar{v}_{k} \in \bar{A}\left(\bar{y}_{k}\right)$ satisfying

$$
\bar{x}_{k}+h \epsilon \bar{v}_{k} \in(1-\delta h) \mathfrak{M} .
$$

Let $\bar{x} \in \mathbb{R}^{n}$. Consider the set-valued maps

$$
\Theta(\bar{x})=\left\{\left(\theta_{1}, \ldots, \theta_{m}\right) \mid \sum_{k=1}^{m} \theta_{k} \bar{x}_{k}=\bar{x}, \sum_{k=1}^{m} \theta_{k}=\mu(\bar{x}), \theta_{k} \geqslant 0\right\},
$$

and

$$
\bar{U}(\bar{x})=\left\{\bar{v}=\sum_{k=1}^{m} \theta_{k} \bar{v}_{k} \mid\left(\theta_{1}, \ldots, \theta_{m}\right) \in \Theta(\bar{x})\right\},
$$

where $\mu(\bar{x})$ is the Minkowski function of $\mathfrak{M}$. We will need the following lemma.

Lemma 3.1. The set-valued map $\bar{U}(\cdot)$ is positively homogeneous, has compact convex images and is upper semi-continuous.

Proof. Let $\bar{x} \in \mathbb{R}^{n}$ and $\alpha>0$. Obviously we have

$$
\alpha \Theta(\bar{x})=\Theta(\alpha \bar{x}),
$$

i.e, $\bar{U}(\cdot)$ is positively homogeneous. Let $\bar{v}^{1}, \bar{v}^{2} \in \bar{U}(\bar{x})$ and $\lambda \in[0,1]$, then we have

$$
\lambda \bar{v}^{1}+(1-\lambda) \bar{v}^{2}=\sum_{k=1}^{m}\left(\lambda \theta_{k}^{1}+(1-\lambda) \theta_{k}^{2}\right) \bar{v}_{k},
$$

where $\sum_{k=1}^{m} \theta_{k}^{j} \bar{x}_{k}=\bar{x}, \sum_{k=1}^{m} \theta_{k}^{j}=\mu(\bar{x})$, and $\theta_{k}^{j} \geqslant 0, j=1$, 2. Since

$$
\begin{aligned}
\lambda \theta_{k}^{1}+(1-\lambda) \theta_{k}^{2} & \geqslant 0, \quad \lambda \in[0,1], \\
\sum_{k=1}^{m}\left(\lambda \theta_{k}^{1}+(1-\lambda) \theta_{k}^{2}\right) \bar{x}_{k} & =\lambda \bar{x}+(1-\lambda) \bar{x}=\bar{x}, \\
\sum_{k=1}^{m}\left(\lambda \theta_{k}^{1}+(1-\lambda) \theta_{k}^{2}\right) & =\lambda \mu(\bar{x})+(1-\lambda) \mu(\bar{x})=\mu(\bar{x}),
\end{aligned}
$$


we get $\lambda \bar{v}^{1}+(1-\lambda) \bar{v}^{2} \in \bar{U}(\bar{x})$, i.e., the set-valued map has convex images.

If $\bar{v}^{i} \in \bar{U}\left(\bar{x}^{i}\right), \sum_{k=1}^{m} \theta_{k}^{i} \bar{x}_{k}=\bar{x}^{i}, \sum_{k=1}^{m} \theta_{k}^{i}=\mu\left(\bar{x}^{i}\right), \theta_{k}^{i} \geqslant 0$, and $\bar{x}^{i} \rightarrow \bar{x}$, $\bar{v}^{i} \rightarrow \bar{v}$ as $i \rightarrow \infty$, then, without loss of generality, $\theta_{k}^{i} \rightarrow \theta_{k}$. Since $\mu(\cdot)$ is Lipschitzian, passing to the limit as $i \rightarrow \infty$, we obtain

$$
\sum_{k=1}^{m} \theta_{k} \bar{x}_{k}=\bar{x}, \sum_{k=1}^{m} \theta_{k}=\mu(\bar{x}), \theta_{k} \geqslant 0 .
$$

Therefore the graph of $\bar{U}(\cdot)$ is closed. Since $\bar{U}(\cdot)$ is a bounded map, this means that $\bar{U}(\cdot)$ is upper semi-continuous.

We are now in position to state and prove our main result.

Theorem 3.2. Let $A: \mathbb{R} \times \mathbb{R}^{n} \rightarrow \mathcal{F}\left(\mathbb{R}^{n}\right)$ be a convex process satisfying conditions (C5) - (C6). Suppose that $\bar{x}=0$ is a weakly asymptotically stable equilibrium position of inclusion (3.2). Then, the origin is a weakly exponentially stable equilibrium position of differential inclusion (3.1).

Proof. Consider the polyhedron $\mathfrak{M}=\operatorname{co}\left\{\bar{x}_{k}\right\}$, introduced above. Recall that there exist $h>0$ and $\delta \in[0,1$ [ such that for every $k$, there is a vector $\bar{v}_{k} \in \bar{A}\left(\bar{x}_{k}\right)$, satisfying

$$
\bar{x}_{k}+h \epsilon \bar{v}_{k} \in(1-\delta h) \mathfrak{M} .
$$

If $\gamma>0$ sufficiently small then we have

$$
\bar{x}_{k}+h \epsilon\left(\bar{v}_{k}+\gamma \bar{x}_{k}\right) \in\left(1-\frac{\delta h}{2}\right) \mathfrak{M} .
$$

for all $k=\overline{1, m}$. Consider the differential inclusion

$$
\frac{d \bar{z}}{d t} \in \epsilon(\bar{U}(\bar{z})+\gamma \bar{z})
$$

where the set-valued map $\bar{U}(\cdot)$ is defined by equality $(3.3)$. Let $\bar{z}(\cdot)$ be a solution to inclusion (3.5). The function $\mu(\bar{z}(t))$ is absolutely continuous. Let $t$ be a point such that the derivatives

$$
\frac{d \mu(\bar{z}(t))}{d t} \text { and } \frac{d \bar{z}(t)}{d t} \in \epsilon(\bar{U}(\bar{z})+\gamma \bar{z})
$$

exist. Then, there exists $\bar{v}(t) \in \bar{U}(\bar{z}(t))$ such that

$$
\frac{d \bar{z}(t)}{d t}=\epsilon(\bar{v}(t)+\gamma \bar{z}(t))
$$

Moreover, we have

$$
\bar{z}(t)+h \epsilon(\bar{v}(t)+\gamma \bar{z}(t))=\bar{z}(t)+h \epsilon\left(\sum_{k=1}^{m} \theta_{k} \bar{v}_{k}+\gamma \bar{z}(t)\right),
$$

where $\left(\theta_{1}, \ldots, \theta_{m}\right) \in \Theta(\bar{z}(t))$. Therefore we obtain

$$
\begin{gathered}
\bar{z}(t)+h \epsilon(\bar{v}(t)+\gamma \bar{z}(t))= \\
=\sum_{k=1}^{m} \theta_{k}\left(\bar{x}_{k}+h \epsilon\left(\bar{v}_{k}+\gamma \bar{x}_{k}\right)\right) \in\left(1-\frac{\delta h}{2}\right) \mu(\bar{z}(t)) \mathfrak{M} .
\end{gathered}
$$


From this we get

$$
\begin{aligned}
\frac{d}{d t} \mu(\bar{z}(t)) & \leqslant \frac{\mu(\bar{z}(t)+h \epsilon(\bar{v}(t)+\gamma \bar{z}(t)))-\mu(\bar{z}(t))}{h} \\
& \leqslant \frac{\left(1-\frac{\delta h}{2}\right) \mu(\bar{z}(t))-\mu(\bar{z}(t))}{h} \\
& =-\frac{\delta}{2} \mu(\bar{z}(t)) .
\end{aligned}
$$

Thus we have

$$
\mu(\bar{z}(t)) \leqslant \mu(\bar{z}(0)) e^{-\frac{\delta t}{2}}
$$

This implies that $\bar{z}=0$ is an asymptotically stable equilibrium position of inclusion (3.5). Consider the differential inclusion

$$
\frac{d z(t)}{d t} \in \epsilon(W(t, z(t))+\gamma z(t))
$$

where

$$
W(t, z)=\left\{w=\sum_{k=1}^{m} \theta_{k} a_{k}\left(t, \bar{x}_{k}\right) \mid\left(\theta_{1}, \ldots, \theta_{m}\right) \in \Theta(z)\right\},
$$

and the functions $a_{k}\left(t, \bar{x}_{k}\right) \in A\left(t, \bar{x}_{k}\right), k=\overline{1, m}$, satisfy

$$
\bar{v}_{k}=\frac{1}{T} \int_{0}^{T} a_{k}\left(t, \bar{x}_{k}\right) d t .
$$

As in Lemma 3.1, one can show that the set $W(t, z)$ is compact and convex for all $(z, t)$ and the set-valued map $W(t, \cdot)$ is upper semi-continuous. Moreover, conditions $(H 1)-(H 5)$ are satisfied. By Theorem 2.4, there exists a number $\epsilon_{0}>0$ such that the solutions of inclusion (3.7) are bounded, whenever $\epsilon \in] 0, \epsilon_{0}[$ and the initial conditions are sufficiently close to zero. Moreover, from the homogeneity of the right-hand side of inclusion (3.7) we see that there exists a constant $c>0$ such that

$$
|z(t)| \leqslant c|z(0)|, \quad t \geqslant 0
$$

Let $z(\cdot)$ be a solution of inclusion (3.7). Set $z(t)=e^{\gamma \epsilon t} x(t)$. Then we get

On the other hand, we have

$$
\frac{d z(t)}{d t}=\gamma \epsilon e^{\gamma \epsilon t} x(t)+e^{\gamma \epsilon t} \frac{d x(t)}{d t} .
$$

$$
\frac{d z(t)}{d t}=\epsilon\left(\sum_{k=1}^{m} \theta_{k}(t) a_{k}\left(t, \bar{x}_{k}\right)+\gamma z(t)\right)
$$

where $\left(\theta_{1}(t), \ldots, \theta_{m}(t)\right) \in \Theta(z(t))$. From (3.4) we obtain

$$
\begin{aligned}
\frac{d x(t)}{d t} & =\epsilon \sum_{k=1}^{m} e^{-\epsilon \gamma t} \theta_{k}(t) a_{k}\left(t, \bar{x}_{k}\right) \\
& =\epsilon \sum_{k=1}^{m} \tilde{\theta}_{k}(t) a_{k}\left(t, \bar{x}_{k}\right)
\end{aligned}
$$


where $\left(\tilde{\theta}_{1}(t), \ldots, \tilde{\theta}_{m}(t)\right) \in \Theta(x(t))$. From (3.8) we see that the solutions of equation (3.9) satisfy the inequality

$$
|x(t)| \leqslant \tilde{c}|x(0)| e^{-\gamma \epsilon t}
$$

i.e., $x=0$ is a weakly exponentially stable equilibrium position of inclusion

$$
\dot{x} \in \epsilon A(t, x) \text {. }
$$

From Theorem 2.3 we obtain the result.

\section{Differential inclusions generated by control systems}

The obtained result can be applied to differential inclusions generated by control systems. Consider the following control system

$$
\dot{x}=f(t, x, u), u \in U \subset \mathbb{R}^{m},
$$

where $f: \mathbb{R} \times \mathbb{R}^{n} \times U \rightarrow \mathbb{R}^{n}$ is a function satisfying the following conditions:

(M1) There exists a periodic control $u_{0}: \mathbb{R} \rightarrow U$ of period $T$ such that $f\left(0, t, u_{0}(t)\right)=0$ for all $t$

(M2) The function $f$ is periodic in $t$ with the period $T>0$, i.e., $f(t+T, x, u)=$ $f(t, x, u)$ for all $(t, x, u) \in \mathbb{R} \times \mathbb{R}^{n} \times U$

(M3) The function $f$ is continuous in $(x, u)$, measurable in $t$ for all $(x, u) \in$ $\mathbb{R}^{n} \times U$, and $f(t, x, u) \in b(t) B_{n}$ for all $(t, x, u) \in[0, T] \times \mathbb{R}^{n} \times U$, where $b(\cdot) \in L_{1}([0, T], \mathbb{R}) ;$

(M4) The function $f$ is differentiable with respect to $x$ and its gradient is bounded, namely $\left|\nabla_{x} f(t, x, u)\right| \leqslant k(t)$ for all $(t, x, u) \in[0, T] \times \mathbb{R}^{n} \times U$, where $k(\cdot) \in L_{1}([0, T], \mathbb{R})$;

(M5) The set $f(t, x, U)$ is compact for all $(t, x) \in \mathbb{R} \times \mathbb{R}^{n}$.

We can associate the control system (4.1) to the differential inclusion

$$
\dot{x} \in F(t, x) \equiv f(t, x, U)=\bigcup_{u \in U} f(t, x, u) .
$$

It can be shown that the set-valued map $A(t, x)=C(t) x+K(t)$, with $C(t)=\nabla_{x} f\left(t, 0, u_{0}\right)$ and $K(t)=\mathrm{cl} \bigcup_{\alpha>0} \alpha \operatorname{cof}(t, 0, U)$, is a first approximation of $\operatorname{co} F(t, x)$, see [13, Proposition 2.8].

Set $\bar{C}=\frac{1}{T} \int_{0}^{T} C(s) x d s$ and

$$
\bar{K}=\left\{\frac{1}{T} \int_{0}^{T} u(s) d s \mid u(s) \in K(t), u(t) \in L_{1}\left([0, T], \mathbb{R}^{n}\right)\right\} .
$$

Consider the averaged first approximation to inclusion (4.2)

$$
\dot{\bar{x}} \in \bar{C} x+\bar{K}
$$

From Theorem 3.2, we obtain the following result. 
Theorem 4.1. Let $f: \mathbb{R} \times \mathbb{R}^{n} \times U \rightarrow \mathbb{R}^{n}$ be a function satisfying conditions (M1) - (M5). Suppose that $\bar{x}=0$ is a weakly exponentially stable equilibrium position of inclusion (4.3). Then, the origin is weakly exponentially stable equilibrium position of differential inclusion (4.2).

We finish the section with an illustrative example.

Example. Consider the control system

$$
\ddot{x}=-\sin (x)+u \sin \left(\frac{t}{\epsilon}\right),
$$

where $\epsilon>0$ is a small parameter and $u$ is a control subject to the constrain $u \geq 0$. Rewriting the system we have

$$
\left\{\begin{array}{l}
\dot{x}=v \\
\dot{v}=-\sin (x)+u \sin \left(\frac{t}{\epsilon}\right)
\end{array}\right.
$$

Set $\tau=t / \epsilon, y(\tau)=x(\epsilon \tau)$ and $w(\tau)=v(\epsilon \tau)$. Then (4.4) takes the form

$$
\left\{\begin{array}{rl}
d y / d \tau & =\epsilon w \\
d w / d \tau & =-\epsilon \sin (y)+\epsilon u \sin (\tau)
\end{array} .\right.
$$

After linearization at the equilibrium position $(y, w)=(0,0)$, we obtain

$$
\frac{d}{d \tau}\left(\begin{array}{c}
\tilde{y} \\
\tilde{w}
\end{array}\right)=\epsilon\left[\left(\begin{array}{cc}
0 & 1 \\
-1 & 0
\end{array}\right)\left(\begin{array}{c}
\tilde{y} \\
\tilde{w}
\end{array}\right)+\left(\begin{array}{c}
0 \\
\tilde{u} \sin (\tau)
\end{array}\right)\right], \quad \tilde{u} \geqslant 0 .
$$

Obviously we have

$$
\left\{\frac{1}{2 \pi} \int_{0}^{2 \pi} \tilde{u} \sin (\tau) d \tau \mid \tilde{u} \geqslant 0, \tilde{u}(\cdot) \in L_{1}([0,2 \pi], \mathbb{R})\right\}=\mathbb{R} .
$$

Therefore the averaged inclusion is

$$
\frac{d}{d \tau}\left(\begin{array}{c}
\bar{y} \\
\bar{w}
\end{array}\right) \in \epsilon\left[\left(\begin{array}{cc}
0 & 1 \\
-1 & 0
\end{array}\right)\left(\begin{array}{c}
\bar{y} \\
\bar{w}
\end{array}\right)+\left(\begin{array}{c}
0 \\
\mathbb{R}
\end{array}\right)\right] .
$$

This inclusion is weakly asymptotically stable. From Theorem 3.2 we see that the zero equilibrium position of control system (4.4) is weakly exponentially stable.

\section{Acknowledgment}

This research is supported by the Portuguese Foundation for Science and Technologies (FCT), the Portuguese Operational Programme for Competitiveness Factors (COMPETE), the Portuguese Strategic Reference Framework (QREN), the European Regional Development Fund (FEDER) and by the Polytechnic Institute of Viseu through PROFAD scholarship. 


\section{References}

[1] D. Aeyels and J. Peuteman, On exponential stability of nonlinear time-varying differential equations, Automatica, vol. 35, pp. 1091-1100, 1999.

[2] N. N. Bogoliubov and Y. A. Mitropolski, Asymptotic Methods in the Theory of Non-Linear Oscillations, Gordon and Breach, 1961.

[3] V. Burd, Method of Averaging for Differential Equations on an Infinite Interval, Chapman and Hall, 2007.

[4] R. Gama, A. Guerman, G. Smirnov, On the asymptotic stability of discontinuous systems analysed via the averaging method, Nonlinear Analysis: Theory, Methods \& Applications, Volume 74, Issue 4, 15 February 2011, pp. 1513-1522.

[5] R. Gama, G. Smirnov, Exponential Stability via Averaging Method and Applications, In Proceedings of the 1st IAA Conference on Dynamics and Control of Space Systems - DyCoSS2012, Murch, 19-21, 2012, Porto, Portugal (to appear).

[6] G. Grammel, I. Maizurna, Exponential stability and partial averaging, Journal of Mathematical Analysis and Applications, Volume 283, Issue 1, pp. 276286, 2003.

[7] R. M'Closkey, An averaging theorem for time-periodic degree zero homogeneous differential equations, Systems \& Control Letters, Volume 32 Issue 3, Nov. 17, 1997.

[8] P. Morin, C. Samson, Time-varying exponential stabilization of a rigid spacecraft with two control torques, IEEE Transactions on Automatic Control, Volume 42, Issue 4, pp. 528 - 534, 1997.

[9] H. K. Khalil, Nonlinear Systems, Englewood Cliffs, NJ: Prentice Hall, third ed. - 2002.

[10] S. Klymchuk, A. Plotnikov, N. Skripnik, Overview of V.A. Plotnikov's research on averaging of differential inclusions, Physica D: Nonlinear Phenomena, Available online 27 May 2011

[11] J. Peuteman and D. Aeyels, Averaging Results and the Study of Uniform Asymptotic Stability of Homogeneous Differential Equations That Are Not Fast Time-Varying, SIAM J. Control Optim. 37, pp. 997-1010, 1999.

[12] G. V. Smirnov, Weak asymptotic stability at first approximation for periodic differential inclusions, Nonlinear Differential Equations and Applications Volume 2, Number 4, pp. 445-461, 1995.

[13] G. V. Smirnov, Introduction to the Theory of Differential Inclusions, AMS Graduate Studies in Mathematics, Vol 41, 2002.

R. Gama

School of Technology and Management of Lamego

Av. Visconde Guedes Teixeira, 5100-074 Lamego Portugal

e-mail: rgama@estgl.ipv.pt

G. Smirnov

Centre of Physics

Department of Mathematics and Applications, University of Minho

Campus de Gualtar, 4710-057 Braga, Portugal

e-mail: smirnov@math.uminho.pt 